\title{
THE COMPOSITION OF HARMONIC MAPPINGS
}

\author{
EDGAR REICH ${ }^{1}$
}

\section{Introduction}

The function $f(z)$ is said to be a harmonic mapping if $f(z)$ is complex-valued harmonic:

$$
f_{\bar{z} z}=0 .
$$

Special properties of two-dimensional harmonic vectors were first considered by Kneser and Radó [3]. There is now a large literature on the subject. ${ }^{2}$ Analytic functions and affine mappings, $f(z)=\mu z+v \bar{z},(\mu, v$ complex constants) constitute the simplest examples. Evidently, $f(z)$ is a harmonic mapping if and only if

$$
f(z)=A(z)+\overrightarrow{B(z)},
$$

where $A(z), B(z)$ are analytic.

The current contribution is devoted to some elementary facts about compositions which seem to have escaped attention. It is easy to verify that if $f$ and $g$ are harmonic mappings, with domain $g \supset$ range $f$, then $g \circ f$ is not "in general" harmonic. Trivial exceptions occur when $f$ (or its conjugate) is analytic and $g$ an arbitrary harmonic mapping, and when $f$ is an arbitrary harmonic mapping and $g$ is affine. That, however, there also exist non-trivial exceptions follows from an example given by Choquet [1] for which

$$
g(f(z)) \equiv z,
$$

even though neither $f$ nor $g$ are analytic, anti-analytic, or affine. Choquet [1, pp.164 165 ] credits J. Deny with proving that Choquet's example of harmonic mappings $f$, $g$ satisfying $(0.1)$ is essentially unique.

Our object (Theorem 1 and its corollary) is to obtain local descriptions of all harmonic mappings $f$ with the property that $g \circ f$ is harmonic for some non-affine harmonic $g$. The descriptions are local in the sense that they concern sufficiently

1) Work done with support from National Science Foundation Grants MCS-8300248 and INT-8414734.

2) See [2] for a recent bibliography. 
small simply-connected neighborhoods of points where whatever non-constant analytic functions are involved are non-zero. It is perhaps surprising that very explicit descriptions can be obtained. For completeness we have also included a proof of the Choquet-Deny theorem, as [1] does not contain one.

\section{Characterization of harmonic decompositions}

We can assume, without loss of generality, that $f(z)$ has the form

$$
f(z)=z+\overline{B(z)},
$$

where $B(z)$ is analytic, and that $g(w)$ has the form,

$$
g(w)=C(w)+\overline{D(w)},
$$

where $C(w), D(w)$ are analytic functions of $w$. The most general harmonic map has the form $f \circ \Phi$, where $\Phi$ is analytic, and $f$ has form (1.1). The problem is to characterize those functions $B(z)$ for which there exist $C(w), D(w)$, with $C^{\prime \prime}(w)$, $D^{\prime \prime}(w)$ not both vanishing, such that $g \circ f \circ \Phi$ is harmonic. Whether or not $B$ has this property is of course independent of $\Phi$.

Let

$$
G(z)=B^{\prime}(z) .
$$

Calculating the laplacian of $g(f(z))$ we find that a necessary and sufficient condition that $g(f(z))$ is harmonic is that

$$
\overline{G(z)} C^{\prime \prime}(w)=-G(z) \overline{D^{\prime \prime}(w)} \quad(w=f(z)=z+\overline{B(z)}) .
$$

Our basic problem is to characterize the solutions of this functional equation. One solution is obtained when $G(z), C^{\prime \prime}(w)$, and $D^{\prime \prime}(w)$ are appropriately related constants.

From now on assume that $G(z)$ is not a constant ${ }^{3}$. Since (1.3) implies that $\left|C^{\prime \prime}(w)\right|=\left|D^{\prime \prime}(w)\right|$, a necessary condition is that $D^{\prime \prime}(w)=\exp (i s) C^{\prime \prime}(w)$ for some real constant $s$, or

$$
\overline{G(z)} \Psi(w)=G(z) \overline{\Psi(w)}, \quad(w=f(z)=z+\overline{B(z)}),
$$

where

$$
C^{\prime \prime}(w)=-i e^{-(i s / 2)} \Psi(w), \quad D^{\prime \prime}(w)=-i e^{i s / 2} \Psi(w) .
$$

The condition that there exists a non-zero analytic function $\Psi(w)$ satisfying (1.4) is necessary and sufficient on $B(z)$.

$\left.{ }^{3}\right)$ In that case there exists a neighborhood in which $f$ is one-to-one. 
To transform (1.4) into a condition involving only $G$, let

$$
\gamma(w)=G\left(f^{-1}(w)\right), \quad(w=f(z)=z+\overline{B(z)}) .
$$

We observe the following: A necessary and sufficient condition that there exists a non-vanishing analytic function $\Psi(w)$ satisfying (1.4) is that

$$
R(w)=\frac{\gamma_{w \bar{w}}}{\gamma}-\frac{\gamma_{w} \gamma_{\bar{w}}}{\gamma^{2}}=\text { real function of } w .
$$

The condition (1.6) is necessary, because, by (1.4),

$$
\log \overline{\gamma(w)}+\log \Psi(w)=\log \gamma(w)+\log \overline{\Psi(w)},
$$

and if we operate with $\partial^{2} / \partial \bar{w} \partial w$ on both sides we obtain $R(w)=\overline{R(w)}$. On the other hand, if (1.5) holds then

$$
\frac{\partial}{\partial \bar{w}} \cdot\left[\frac{\gamma_{w}}{\gamma}-\frac{\overline{\gamma_{\bar{w}}}}{\bar{\gamma}}\right]=0
$$

Therefore,

and, hence,

$$
\frac{\partial}{\partial w}[\log \gamma-\log \bar{\gamma}]=F_{1}(w)=\text { analytic function of } w,
$$

$$
\log \gamma-\log \bar{\gamma}=\int F_{1}(w) d w-\overline{F_{2}(w)}
$$

where $F_{2}(w)$ is also an analytic function of $w$. Thus,

$$
\frac{\gamma(w)}{\overline{\gamma(w)}}=\frac{L(w)}{\overline{M(w)}}, \quad \text { with } L(w)=\exp \int F_{1}(w) d w, \quad M(w)=\exp F_{2}(w) .
$$

Since it follows that $|L(w)|=|M(w)|$, we have $M(w)=\exp (i t) L(w)$ for some real constant $t$. Setting

$$
\Psi(w)=e^{i t / 2} L(w),
$$

we obtain $\gamma(w) \overline{\Psi(w)}=\overline{\gamma(w)} \Psi(w)$. This shows that (1.6) is also sufficient for (1.4).

The next step is to employ (1.5) to compute $R(f(z))$, making use of the relations

$$
\begin{gathered}
\frac{\partial z}{\partial w}=\frac{\overline{f_{z}}}{J}=\frac{1}{J}, \quad \frac{\partial z}{\partial \bar{w}}=-\frac{f_{\bar{z}}}{J}=-\frac{\bar{G}}{J}, \\
J=\left|f_{z}\right|^{2}-\left|f_{\bar{z}}\right|^{2}=1-|G|^{2}, \quad G=B^{\prime} .
\end{gathered}
$$

We find that

Therefore,

$$
\begin{gathered}
\gamma_{w} \circ f=G^{\prime} / J, \quad \gamma_{\bar{w}} \circ f=-\bar{G} G^{\prime} / J, \\
\gamma_{w \bar{w}} \circ f=\left(-\bar{G}^{2} G^{2}+G\left|G^{\prime}\right|^{2}-J \bar{G} G^{\prime \prime}\right) / J^{3} .
\end{gathered}
$$

$$
J^{3} R(f(z))=-\frac{\bar{G}^{2} G^{\prime 2}}{G}+\left|G^{\prime}\right|^{2}-J \frac{\bar{G} G^{\prime \prime}}{G}+J \frac{\bar{G} G^{2}}{G^{2}} .
$$


This suggests defining

$$
R_{1}(z)=\frac{J^{3} R(f(z))-\left|G^{\prime}\right|^{2}}{|G|^{2}},
$$

which is real if and only if $R(f(z))$ is real. By (1.8),

$$
R_{1}(z)=S(z)-T(z) \overline{G(z)},
$$

where $S, T$ are the following analytic functions:

$$
S(z)=\frac{G^{\prime}(z)^{2}-G(z) G^{\prime \prime}(z)}{G(z)^{3}}, \quad T(z)=\frac{2 G^{\prime}(z)^{2}-G(z) G^{\prime \prime}(z)}{G(z)^{2}} .
$$

Since $R_{1}$ is real we have

$$
S-\bar{S}=\bar{G} T-G \bar{T},
$$

and this condition is equivalent to the basic requirement (1.6). Applying $\partial^{2} / \partial \bar{z} \partial z$ to both sides, one sees that (1.11) holds if and only if

$$
S(z)=a-\bar{\varkappa} G(z), \quad T(z)=c G(z)+\varkappa,
$$

where $a$ and $c$ are real constants and $\chi$ is a complex constant.

Relations (1.10) and (1.12) yield the pair of simultaneous differential equations

$$
G^{2}-G G^{\prime \prime}=a G^{3}-\bar{\varkappa} G^{4}, \quad 2 G^{2}-G G^{\prime \prime}=c G^{3}+\varkappa G^{2}
$$

which constitute necessary and sufficient conditions on $G$. Subtracting the first equation from the second we obtain the equivalent pair

$$
G^{2}=\bar{\varkappa} G^{4}+(c-a) G^{3}+\varkappa G^{2}, \quad G^{2}-G G^{\prime \prime}=a G^{3}-\bar{\chi} G^{4} .
$$

The key to the situation is that the two equations (1.13) are highly dependent. The first implies

$$
\frac{d}{d z}\left(\frac{G^{\prime}}{G}\right)^{2}=[2 \bar{\varkappa} G+(c-a)] G^{\prime}
$$

On the other hand, the second equation of (1.13) implies that

$$
\frac{d}{d z}\left(\frac{G^{\prime}}{G}\right)^{2}=\frac{2 G^{\prime}}{G}\left(\bar{x} G^{2}-a G\right)=2(\bar{x} G-a) G^{\prime} .
$$

So we must have $a=-c$. Conversely, if we set $a=-c$, then we see, as above, that the first of equations (1.13) implies the second.

We note that if $x=c=a=0$, then (1.13) also holds when $G$ is constant. Thus we have proved the following:

Theorem 1. Suppose $f(z)=z+\overline{B(z)}, G(z)=B^{\prime}(z)$. A necessary and sufficient condition that there locally exists a non-affine complex harmonic function $g(w)$, such 
that $g(f(z))$ is harmonic is that $G(z)$ satisfies

$$
G^{\prime 2}=\alpha^{2} G^{4}+2 c G^{3}+\bar{\alpha}^{2} G^{2}
$$

for some complex constant $\alpha$ and some real constant $c$.

$G(z)$ and $B(z)$ can be expressed in terms of elementary functions. We distinguish the following separate cases; the functions $G(z)$ are given up to a translation of the $z$ plane.

$$
\alpha=c=0, \quad G(z)=\text { const. }
$$

$$
\alpha=0, \quad c \neq 0, \quad G(z)=\frac{2}{c z^{2}} \text {. }
$$$$
\alpha \neq 0, \quad c=r|\alpha|^{2}, \quad\left(r=\text { real const, } r^{2} \neq 0,1\right),
$$

$$
G(z)=-\frac{\bar{\alpha} / \alpha}{r+\sqrt{1-r^{2}} \sinh (\bar{\alpha} z)} .
$$

$$
\begin{aligned}
\alpha \neq 0, \quad c=0, \quad G(z) & =-\frac{\bar{\alpha} / \alpha}{\sinh (\bar{\alpha} z)} . \\
\alpha \neq 0, \quad c=|\alpha|^{2}, \quad G(z) & =-\frac{\bar{\alpha}}{\alpha} \frac{e^{\bar{\alpha} z}}{e^{\bar{\alpha} z}-1} .
\end{aligned}
$$

The above expressions are obtained by integrating the differential equation (1.14) and solving for $G(z)$. Integrating the functions $G(z)$, the following classification of harmonic mappings $f(z)$ is obtained.

Corollary. Suppose $f(z)$ is complex harmonic. A necessary and sufficient condition that there locally exists a non-affine complex harmonic function $g(w)$, such that $g(f(z))$ is harmonic, is that $f(z)$ is ${ }^{4}$ one of the following types, where $v$ is a complex constant, and $r$ a real constant.

Type 0: $f(z)=\Phi(z)$, where $\Phi(z)$ is analytic.

Type 1: $f(z)=\Phi(z)+v \overline{\Phi(z)}, \quad v \neq 0$.

Type 2: $f(z)=\Phi(z)+\frac{r}{\Phi(z)}, \quad r \neq 0$.

Type 3: $f(z)=\Phi(z)+v \overline{\tanh ^{-1}\left(\sqrt{1-r^{2}}-r \tanh \frac{\Phi(z)}{v}\right)}, \quad r^{2} \neq 0,1$.

Type 4: $f(z)=\Phi(z)-v \overline{\log \tanh \frac{\Phi(z)}{2 v}}$.

Type 5: $f(z)=\Phi(z)-v \overline{\log \left[\exp \left(\frac{\Phi(z)}{v}\right)-1\right]}$.

4 Up to translations and conjugation of $z$ and $f(z)$. 
The associated non-affine harmonic mappings $g(w)$ can be determined in principle by integrating (1.6). When $f(z)$ is of type 1 , there is an especially simple solution; namely (up to an additive affine function)

$$
g(w)=\bar{v} w^{2}-v \bar{w}^{2} .
$$

\section{Harmonic decompositions of the identity}

Choquet-Deny Theorem. Suppose $f$ is a sense-preserving harmonic homeomorphism and is neither analytic nor affine. A necessary and sufficient condition that $f^{-1}$ is also harmonic is that

$$
f(z)=\frac{\sigma}{\bar{\alpha}} z+\frac{1}{\bar{\alpha}} \log \left[\frac{\mu-e^{-\sigma z}}{\overline{\mu-e^{-\sigma z}}}\right]+\text { const, }
$$

where $\sigma, \alpha, \mu$ are non-zero complex constants, $|\mu|>\sup _{z}\left|e^{-\sigma z}\right|$.

Proof. While one could proceed to test each of the types 1 to 5 of the corollary of Theorem 1 to see which fulfilled the requirement that $f^{-1}$ is also one of these types, it is more efficient to take advantage of the symmetry of the relation

$$
g(f(z))=z
$$

by means of an independent proof. Assuming

$$
w=f(z)=A(z)+\overline{B(z),}
$$

where $A(z), B(z)$ are analytic, and where

$$
J=\left|A^{\prime}\right|^{2}-\left|B^{\prime}\right|^{2}>0, \quad B^{\prime} \neq 0,
$$

in the domain of definition of $f$, we have, in place of (1.7),

$$
g_{w}=\overline{A^{\prime}} / J, \quad g_{\bar{w}}=-\overline{B^{\prime}} / J .
$$

By (2.3), (2.4), (2.5) one finds that $g_{\overline{\mathrm{w}} w}=0$ if and only if

$$
A^{\prime}\left|B^{\prime}\right| \overline{A^{\prime \prime}}-\left|A^{\prime}\right|^{2} B^{\prime} \overline{B^{\prime \prime}}=\overline{A^{\prime 2}} \overline{B^{\prime}} A^{\prime \prime}-\overline{A^{\prime}} \overline{B^{\prime}} B^{\prime \prime} .
$$

Dividing by $\left|A^{\prime}\right|^{2}\left|B^{\prime}\right|^{2}$, (2.6) becomes

$$
\frac{\overline{A^{\prime \prime}}}{\overline{A^{\prime}}}-\frac{\overline{B^{\prime \prime}}}{\overline{B^{\prime}}}=\alpha \overline{A^{\prime}}-\beta \overline{B^{\prime}}, \quad \text { where } \quad \alpha(z)=\frac{A^{\prime \prime}}{A^{\prime} B^{\prime}}, \quad \beta(z)=\frac{B^{\prime \prime}}{A^{\prime} B^{\prime}},
$$

or, equivalently,

By (2.4), therefore,

$$
(\bar{\alpha}+\beta) \overline{B^{\prime}}=(\alpha+\bar{\beta}) \overline{A^{\prime}} .
$$

$$
\beta=-\bar{\alpha} .
$$


Since $\alpha, \beta$ are both analytic, they must therefore both be constants,

$$
A^{\prime \prime}=\alpha A^{\prime} B^{\prime}, \quad B^{\prime \prime}=-\bar{\alpha} A^{\prime} B^{\prime} .
$$

Since affine $f$ are excluded, $\alpha$ cannot be 0 . Eliminating $B$ between the differential equations (2.8),

$$
\frac{B^{\prime \prime}}{B^{\prime}}=-\bar{\alpha} A^{\prime}=\frac{\alpha A^{\prime} B^{\prime \prime}}{A^{\prime \prime}}=\frac{A^{\prime} A^{\prime \prime \prime}-A^{\prime \prime 2}}{A^{\prime} A^{\prime \prime}}=\frac{A^{\prime \prime \prime}}{A^{\prime \prime}}-\frac{A^{\prime \prime}}{A^{\prime}} .
$$

Integrating, we obtain

$$
A^{\prime}=\text { const } e^{-\bar{\alpha} A}+\text { const. }
$$

This is a separable differential equation. For the solution we have

$$
\begin{gathered}
A(z)=\frac{\sigma}{\bar{\alpha}} z+\frac{1}{\bar{\alpha}} \log \left(\mu-e^{-\sigma z}\right)+\text { const, } \\
A^{\prime}(z)=\frac{\mu \sigma}{\bar{\alpha}}\left(\mu-e^{-\sigma z}\right)^{-1}, \quad A^{\prime \prime}(z)=-\frac{\mu \sigma^{2}}{\bar{\alpha}}\left(\mu-e^{-\sigma z}\right)^{-2} e^{-\sigma z} .
\end{gathered}
$$

Therefore, by the first equation of (2.8),

$$
B^{\prime}(z)=-\frac{\sigma}{\alpha} \frac{e^{-\sigma z}}{\mu-e^{-\sigma z}}
$$

and therefore,

$$
B(z)=-\frac{1}{\alpha} \log \left(\mu-e^{-\sigma z}\right)+\text { const. }
$$

Conversely, if (2.9), (2.10) hold then (2.7) is satisfied.

The restriction on $\mu$ in the statement of the theorem is to insure that $J>0$. We note that the harmonic mapping $f$ of the Choquet-Deny Theorem falls within type 5 of Section 1.

\section{References}

[1] CHOQUET, G.: Sur un type de transformation analytique généralisant la représentation conforme et définie au moyen de fonctions harmoniques. - Bull. Sci. Math. 69, 1945, $156-165$.

[2] Eells, J., and L. Lemaire: A report on harmonic maps. - Bull. London Math. Soc. 10, 1978, $1-68$.

[3] KNeSER, H., and T. Radó: Aufgabe 41. - Jber. Deutsch. Math. Verein. 35, 1926, Italic pp. $123-124$.

University of Minnesota

School of Mathematics

Minneapolis, Minnesota 55455

USA

Received 26 November 1985 